\title{
TWO LARGE SUBSETS OF A FUNCTIONAL SPACE
}

\author{
F.S. CATER \\ Department of Mathematics \\ Portland State University \\ Portland, Oregon 97207 U.S.A. \\ (Received May 29, 1984)
}

ABSTRACT. Let $P_{1}$ denote the Banach space composed of all bounded derivatives $f$ of everywhere differentiable functions on $[0,1]$ such that the set of points where $f$ vanishes is dense in $[0,1]$. Let $D_{0}$ consist of those functions in $P_{1}$ that are unsigned on every interval, and let $D_{1}$ consist of those functions in $P_{1}$ that vanish on dense subsets of measure zero. Then $D_{0}$ and $D_{1}$ are dense $G_{\delta}$-subsets of $P_{1}$ with void interior. Neither $D_{0}$ nor $D_{1}$ is a subset of the other.

KEY WORDS AND PHRASES. Banach space of functions, derivative dense $C_{\delta}$-nub.et. 1980 MATHFMATICS SUBJECT CLASSJFICATION: $26 A 24$

1. INTRODUCTION.

The real vector space $D$ of all bounded derivatives of everywhere differentiable functions on $[0,1]$ is a Banach space [1] under the norm

$$
\|\mathrm{f}\|=\sup _{0 \leq x \leq 1}|f(x)| .
$$

(At the endpoints 0 and 1 we require that the one sided derivatives exist.) Tibor Salat essentially proved [1] that the set

$$
D_{0}=\{f \in D: \quad f \text { is unsigned on any interval }\}
$$

is a nowhere dense subset of D. To do this, he observed that

$$
P_{1}=\{f \in D: f=0 \text { on a dense subset of }[0,1]\}
$$

is a nowhere dense Banach subspace of $D$ and $D_{0}<P_{1}$. Since $P_{1}$ is a Banach space in its own right, it is natural to study $D_{0}$ as a subset of $P_{1}$. Put

$$
D_{1}=\left\{f \in P_{1}: f \neq 0 \text { almost everywhere on }[0,1]\right\}
$$

In this note, we prove that $D_{0}$ and $D_{1}$ are "large" subsets of the Banach space $P_{1}$. THEOREM 1. $\mathrm{D}_{0}$ is a dense $\mathrm{G}_{\delta}$-set in $\mathrm{P}_{1}$ with vold interior. THEOREM 2. $D_{1}$ is a dense $G_{\delta}$-set in $P_{1}$ with void interior. 
Now put $\quad E=\left\{f \in P_{1}: f\right.$ is not almost everywhere discontinous $\}$.

If $f \in P_{1}$, then $f$ must vanish at every point where $f$ is continuous, and $E \subset P_{1} \backslash D_{1}$. We obtain from Theorem 2, a result of Clifford Weil [2].

COROLLARY 1 (C. Weil). $E$ is a first category subset of $P_{1}$.

Clifford Weil [3] proved most of Theorem 1. Finally, we show that neither of the sets $D_{0}$ or $D_{1}$ is a subset of the other, and we prove an analogue of Theorem 2 for spaces of nonnegative derivatives. (I take this opportunity to thank the referee for simplifying many of my arguments.)

Proof of Theorem 1. The proof that $D_{0}$ is a dense $G_{\delta}$-set in $P_{1}$ is essentially given in [3], so we leave it.

It remains to prove that $D_{0}$ has void interior. Let $f \in P_{1}$ and $\varepsilon>0$. It is easy to find an interval $[a, b]$ for which $f(a)=f(b)=0$ and $|f(x)|<\varepsilon$ for $x \in[a, b]$. Now let $g(x)=f(x)$ if $x \notin[a, b]$, and $g(x)=0$ if $x \in[a, b]$. clearly $g \in P_{1}$ but $g \notin\left(D_{0} \cup D_{1}\right)$. Finally, $\|\mathrm{f}-\mathrm{g}\| \leq \varepsilon$. Thus every open set in $P_{1}$ contains functions $\& D_{0} \cup D_{1}$, so $D_{0} \cup D_{1}$ has void interior in $P_{1}$.

Proof of Theorem 2. For each positive integer $n$, define

$$
E_{n}=\left\{f \in P_{1}: m\{x: f(x)=0\} \geq n^{-1}\right\}
$$

Then $D_{1}=P_{1} \backslash U_{n} E_{n}$. We claim that each $E_{n}$ is closed in $P_{1}$. Let $f_{k} \in E_{n}$ and $\mathrm{f} \in \mathrm{P}_{1}$ and $\lim _{\mathrm{k} \rightarrow \infty}\left\|\mathrm{f}_{\mathrm{k}}-\mathrm{f}\right\|=0$. Say

$$
A_{k}=\left\{x: f_{k}(x)=0\right\}
$$

and $\mathrm{mA}_{k} \geq \mathrm{n}^{-1}$. Then at each $x \in A=\cap_{j} \cup_{k \geq j} A_{k}, f(x)=0$. But $m A \geq n^{-1}$ so $f \in E_{n}$ Thus $E_{n}$ is closed in $P_{1}$.

It remains to prove that $E_{n}$ is nowhere dense. Let $f \in E_{n}$ and $\varepsilon>0$. Use [4] to get a function $g \in D_{1}$ such that $0 \leq \mathrm{g} \leq 1$. But there is a number $c>0$ such that $c<\varepsilon$ and $m\{x: 0<|f(x)| \leq c\}<n^{-1}$. It follows that

$$
\mathrm{m}\{\mathrm{x}: \mathrm{f}(\mathrm{x})=-\mathrm{cg}(\mathrm{x})\}<\mathrm{n}^{-1} \text {. }
$$

Fina11y,

$$
\mathrm{m}\{\mathrm{x}: \mathrm{f}(\mathrm{x})+\mathrm{cg}(\mathrm{x})=0\}<\mathrm{n}^{-1}
$$

and $\mathrm{f}+\mathrm{cg} \notin \mathrm{E}_{\mathrm{n}}$. Moreover, $\|(\mathrm{f}+\mathrm{cg})-\mathrm{f}\|=\|\mathrm{cg}\| \leq \mathrm{c}<\varepsilon$. Thus $\mathrm{E}_{\mathrm{n}}$ is nowhere dense.

In the proof of Theorem 1 we saw that $D_{0} \cup D_{1}$, and hence $D_{1}$, has void interior.

It follows from Theorems 1 and 2 that $D_{0} \cap D_{1}$, the set of all functions in $P_{1}$ that vanish on dense sets of measure zero and are unsigned in any interval, is a dense $G_{\delta}$-subset of $P_{1}$. Next we show that the sets $D_{0}$ and $D_{1}$ are quite different. Neither is a subset of the other.

THEOREM 3. The Sets $D_{0} \backslash D_{1}$ and $D_{1} \backslash D_{0}$ are nonvoid.

PROOF. Let $h$ be a function in $D_{0}$. We construct a sequence of intervals $\left(a_{n}, b_{n}\right)$, with mutually disjoint closures, such that $b_{n}-a_{n}<2^{-n}$ and $h\left(a_{n}\right)=h\left(b_{n}\right)=0$ for each $n$, and $! !_{n}\left(a_{n}, h_{n}\right)$ is dense in $[0,1]$. Let $h_{n}=h \psi\left(a_{n}, b_{n}\right)$ where $\psi$ means 
characteristic function: for $0 \leq x \leq 1, h_{n}(x)=h(x) \psi_{\left(a_{n}, b_{n}\right)}(x)$. It follows that $h_{n} \in P_{1}$, and the sequence $\left(\left\|h_{n}\right\|\right)$ is bounded. Put

$$
f=\sum_{n} 2^{-n} h_{n} \in P_{1}
$$

Now $f$ cannot be signed on any subinterval of an $\left(a_{n}, b_{n}\right)$, so $f \in D_{0}$. Clearly for $x \in[0,1] \backslash \cup_{n}\left(a_{n}, b_{n}\right), h_{n}(x)=0$ for a11 $n$ and $f(x)=0$. But

$$
m\left\{[0,1] \backslash \cup_{n}\left(a_{n}, b_{n}\right)\right\}=1-\sum_{n}\left(b_{n}-a_{n}\right)>0
$$

Thus $f \in D_{0} \backslash D_{1}$.

We use [4] to obtain a function in $D_{1} \backslash D_{0}$.

Now put

$$
\begin{aligned}
& \mathrm{P}_{2}=\left\{\mathrm{f} \in \mathrm{P}_{1}: \mathrm{f} \text { is nonnegative }\right\} \\
& \mathrm{D}_{2}=\left\{\mathrm{f} \in \mathrm{P}_{2}: \mathrm{f}>0 \text { almost everywhere on }[0,1]\right\} .
\end{aligned}
$$

Then $\mathrm{P}_{2}$ is a complete metric space in its own right. We conclude by showing that $\mathrm{D}_{2}$ is a "large" subset of $\mathrm{P}_{2}$.

THEOREM 4. $\mathrm{D}_{2}$ is a dense $G_{\delta}$-subset of $P_{2}$ with void interior.

PROOF. Define $E_{n}$ as in the proof of Theorem 2. Then $E_{n} \cap P_{2}$ is a closed subset of $P_{2}$. It remains to prove that $D_{2}=P_{2} \backslash \cup_{n}\left(E_{n} \cap P_{2}\right)$ is dense in $P_{2}$. We use [4] to construct $g \in D_{2}$ such that $0 \leq \mathrm{g} \leq 1$. For any $f \in P_{2}$ and any $c>0$, we have $\mathrm{f}+\mathrm{cg} \in \mathrm{D}_{2}$ and $\|(\mathrm{f}+\mathrm{cg})-\mathrm{f}\|=\|\mathrm{cg}\| \leq \mathrm{c}$. So $\mathrm{D}_{2}$ is a dense $\mathrm{G}_{\delta}$-subset of $\mathrm{P}_{2}$

That $D_{2}$ has void interior follows from the same proof (for Theorem 1) that $D_{0}$ has void interior, so we leave this point.

Compare Theorem 4 to the work in [5]. There it is shown that the singular functions form a dense $G_{\delta}$-subset of the complete metric space of continuous nondecreasing functions on $[0,1]$ under the sup metric. When the primitives of the functions in $P_{2}$ are taken, [5] suggests that $D_{2}$ is a "small" subset of $P_{2}$. Of course the metric used in [5] was different from the one used here.

\section{REFERENCES}

1. SALAT, TIBOR, On functions that are monotone on no interval, Amer. Math. Monthly 88 (1981) 754-755.

2. WEIL, CLIFFORD, The space of bounded derivativer, Real Analysis Exchange 3 \#1 (1977-8) 38-41.

3. WEIL, CLIFFORD, On nowhere monotone functions, Proc. Amer. Math. Society, 56 (1976) 388-398.

4. ZAHORSKI, Z., Sur la prémière derivée, Transactions of the Amer. Math. Society, Vol 69 (1950), 26, Lemma 11.

5. ZAMFIRESCU, TUDOR, Most monotone functions are singular, Amer. Math. Monthly 88 (1981) 47-49. 


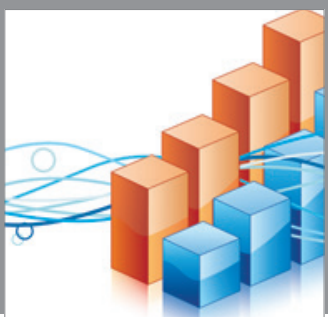

Advances in

Operations Research

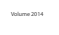

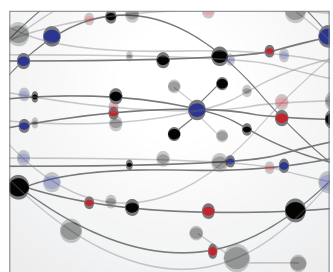

\section{The Scientific} World Journal
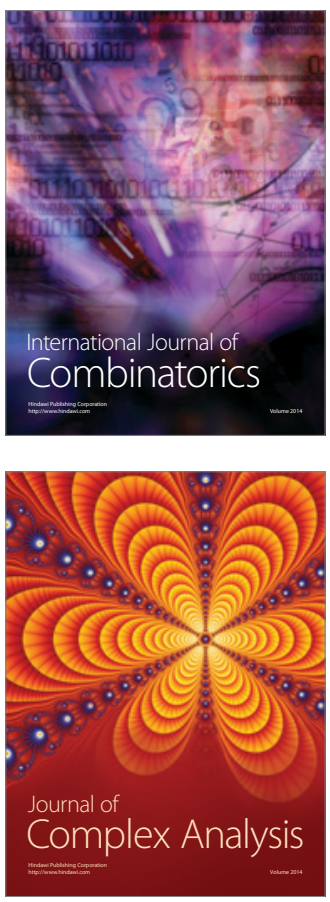

International Journal of

Mathematics and

Mathematical

Sciences
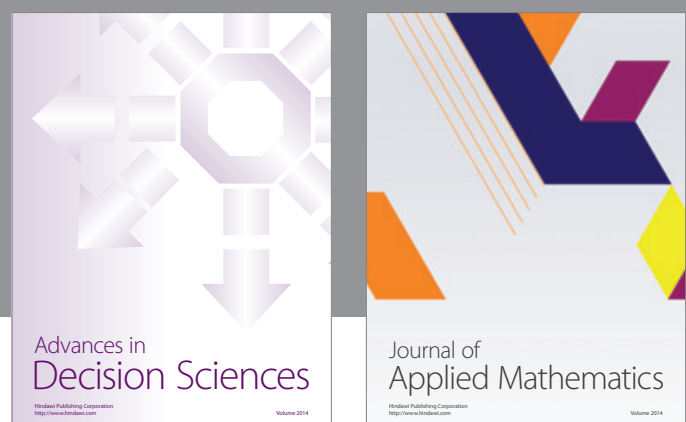

Journal of

Applied Mathematics
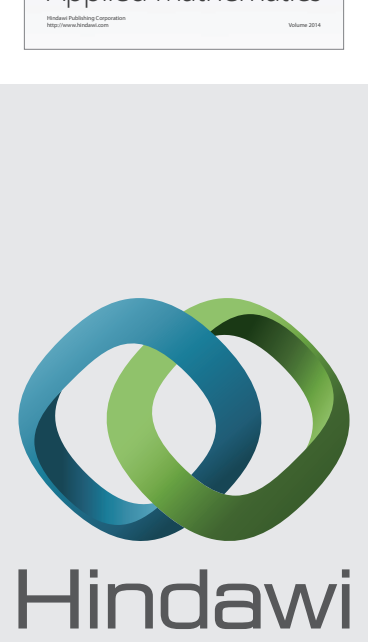

Submit your manuscripts at http://www.hindawi.com
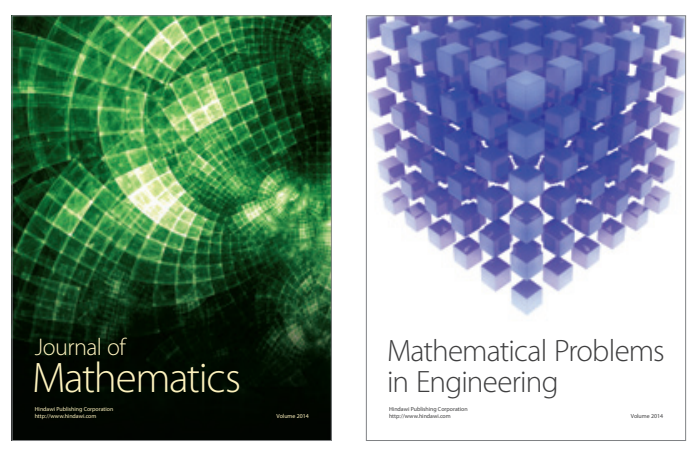

Mathematical Problems in Engineering
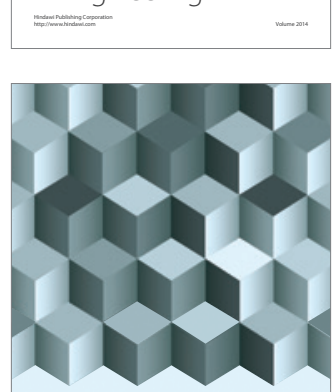

Journal of

Function Spaces
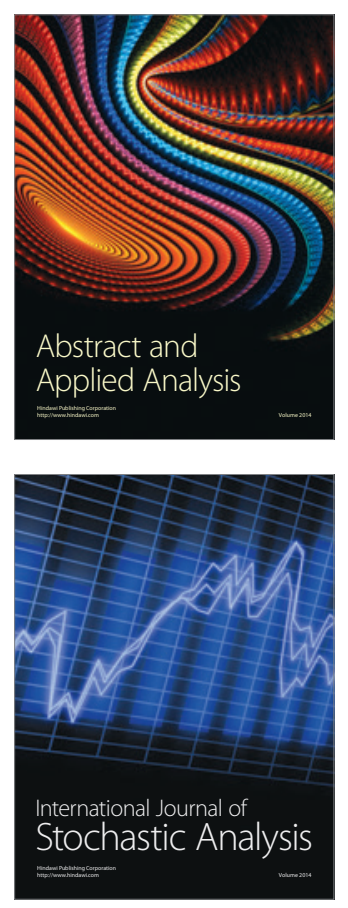

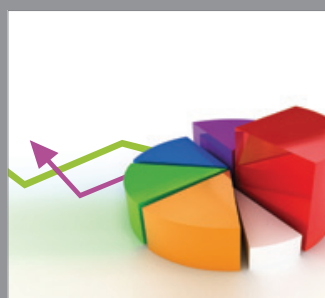

ournal of

Probability and Statistics

Promensencen
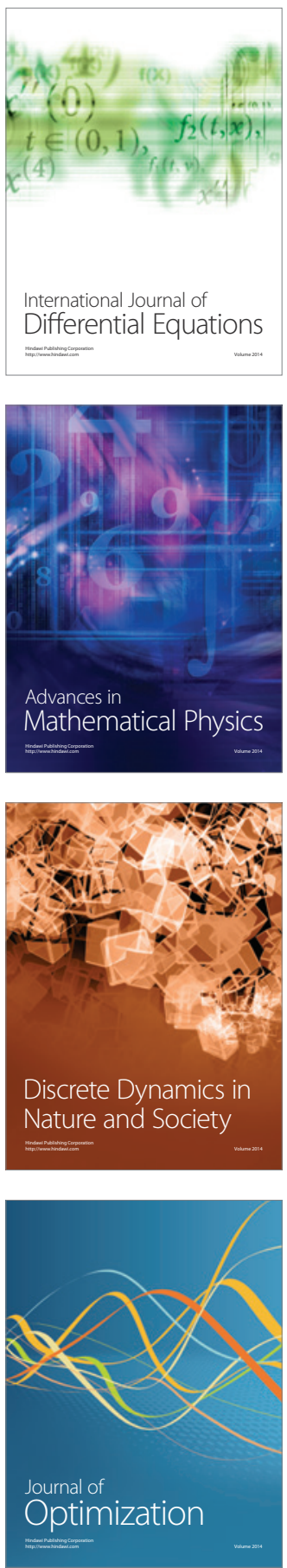